\title{
Design, synthesis, heme binding and density functional theory studies of isoindoline-dione-4-aminoquinolines as potential antiplasmodials
}

Anu Rani ${ }^{1}$, Sumit Kumar ${ }^{1}$, Jenny Legac², Adebayo A Adeniyi ${ }^{3,4}$ iD, Paul Awolade ${ }^{5}$, Parvesh

Singh $^{5}$, Philip J Rosenthal ${ }^{2}$ \& Vipan Kumar*,1

1 Department of Chemistry, Guru Nanak Dev University, Amritsar 143005, Punjab, India

${ }^{2}$ Department of Medicine, University of California, San Francisco, CA 94143, USA

${ }^{3}$ Department of Pharmaceutical Chemistry, University of KwaZulu-Natal, Durban 4000, South Africa

${ }^{4}$ Department of Industrial Chemistry, Federal University of Oye-Ekiti, Nigeria

${ }^{5}$ School of Chemistry \& Physics, University of KwaZulu-Natal, Westville, Durban 4000, South Africa

*Author for correspondence: email:vipan_org@yahoo.com

Aim: WHO Malaria report 2017 estimated 216 million cases of malaria and 445,000 deaths worldwide, with $91 \%$ of deaths affecting the African region. Results/methodology: Microwave promoted the synthesis of cycloalkyl amine substituted isoindoline-1,3-dione-4-aminoquinolines was urbanized for evaluating their antiplasmodial activities. Compound with the optimum combination of propyl chain length and hydroxyethyl piperazine proved to be the most potent among the synthesized scaffolds against chloroquineresistant W2 strain of Plasmodium falciparum with an $\mathrm{IC}_{50}$ value of $0.006 \mu \mathrm{M}$. Heme-binding along with density functional theory studies were further carried out in order to delineate the mechanism of action of the most active compound. Conclusion: The synthesized scaffold can act as a therapeutic template for further synthetic modifications toward the search for a new antimalarial agent.

\section{Graphical abstract:}
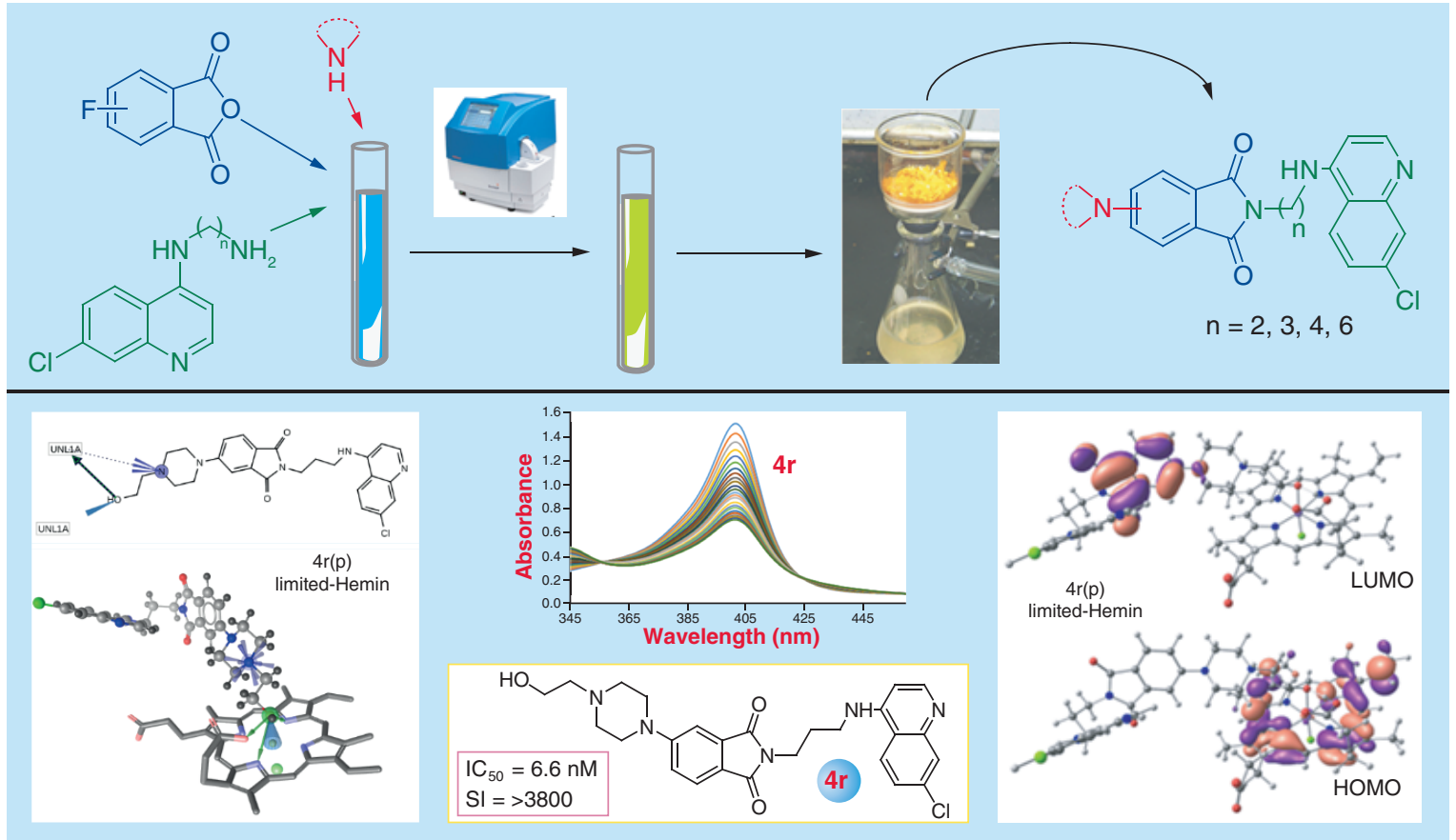

First draft submitted: 6 September 2019; Accepted for publication: 14 November 2019; Published online: 5 December 2019 
Keywords: Antiplasmodial • cycloalkyl-amine • cytotoxicity • density functional theory • heme-binding studies • Isoindoline-1,3-dione-4-aminoquinoline • microwave

Malaria, a life-threatening disease, is transmitted by mosquitoes infected with Plasmodium protozoa [1-3]. Among different species of plasmodia, Plasmodium falciparum is responsible for the most fatal form of malaria, accounting for over $90 \%$ of malaria-related deaths globally [4,5]. There have been significant decreases in malaria morbidity and mortality in recent years, but advances appear to have stalled [6]. The 2018 report on malaria by the World Health Organization estimated 219 million cases of this disease in 2017. The top ten highest afflicted African countries witnessed an increase of 3.5 million cases in 2017 compared with the previous year. Malaria claimed an estimated 435,000 lives in 2017, largely in Africa [7]. In India, the burden of malaria is enormous. The country has the third-highest number of cases in the world and accounts for the highest malaria burden outside of Africa [6].

Chloroquine (CQ) was the drug of choice for the treatment of falciparum malaria for many decades due to its safety, efficacy and low cost [8]. It remains a drug of choice against uncomplicated malaria caused by Plasmodium vivax [9]. In P. falciparum, the development of resistance to CQ necessitated other therapies and paved the way for the development of artemisinin-based combination therapies, the gold standard for the treatment of falciparum malaria [10]. However, the emergence of artemisinin-tolerant $P$. falciparum strains in South-East Asia [11] and recently in Eastern India [12,13] is of serious concern. Therefore, novel strategies to address the challenge of antimalarial drug resistance with the development of new drugs are urgently needed [14-18].

Incorporation of bioactive functionalities in the side chain of 4-aminoquinolines has emerged as a promising strategy to afford enhanced activity against drug-resistant $P$. falciparum [19-22]. Isoindoline-1,3-dione is an important heterocyclic core, with a tendency to potentiate the activities of known antimalarials [23]. Rathi et al. recently reported the in vitro antimalarial activity of a series of isoindoline-1,3-diones functionalized with cyclic amines. Among them, the compound having a piperidinopiperidine group exhibited promising antimalarial activity with an $\mathrm{IC}_{50}$ of $1.2 \mu \mathrm{M}$ [24]. More recently [25], we introduced an isoindoline ring system in the side chain of 4aminochloroquine to enhance activity. These analogs showed remarkable activity against CQ-resistant (CQR) $P$. falciparum. The compound bearing tetrabromo substituted isoindoline ring exhibited the highest potency $\left(\mathrm{IC}_{50}\right.$ $0.1 \mu \mathrm{M}$ ) with low-cytotoxicity toward the normal cells (Figure 1).

Encouraged with these results and recent disclosures from our lab [26-29], we envisaged the incorporation of isoindoline-1,3-diones having cycloalkyl amine functionality at C4/C5 position in the side chain of 4aminoquinolines in order to ascertain their structure-antiplasmodial activity relationship. The introduction of a basic amino substituent is generally considered essential for trapping high concentrations of the drug in the acidic food vacuole of the malaria parasite [30]. The alkyl chain length along with the nature of secondary-amine at C4/C5 position of isoindoline-1,3-diones was varied in order to study the structure-activity relationship (SAR) of the target molecules. Furthermore, heme-binding studies were performed through UV-visible absorption, mass spectrometry and ${ }^{1} \mathrm{H}-\mathrm{NMR}$ titrations of the most potent derivative to gain insights about the binding mode and interactions of the compounds. ClogP values of the neutral and protonated structures of the compounds were calculated to determine their bioavailability. The computational method like density functional theory was performed to govern interactions of compounds with heme.

\section{Materials \& methods \\ General}

Melting points were determined by open capillary using a Veego Precision Digital Melting Point apparatus (MPD) and are uncorrected. ${ }^{1} \mathrm{H}$ NMR spectra were recorded in $\mathrm{CDCl}_{3}$ with Bruker $500(500 \mathrm{MHz})$ and Jeol 400 (400 MHz) spectrometers using tetramethylsilane (TMS) as an internal standard. Microwave reactions were carried out in a Biotage ${ }^{\circledR}$ Initiator + instrument using sealed $2-5 \mathrm{ml}$ process vials. Reaction times refer to irradiation time at the target temperature, not the total irradiation time. The temperature was measured with an infra-red (IR) sensor. Chemical shift values are expressed as parts per million downfield from TMS, and Jvalues are in Hertz. Splitting patterns are indicated as s: singlet, d: doublet, t: triplet, m: multiplet, dd: double doublet, ddd: doublet of a doublet of a doublet and br: broad peak. ${ }^{13} \mathrm{C}$ NMR spectra were recorded on Bruker $125 \mathrm{MHz}$ and Jeol $100 \mathrm{MHz}$ spectrometers in DMSO- $\mathrm{d}_{6}$ using TMS as an internal standard. High-resolution mass spectra were recorded on a Bruker-micrOTOF-Q II spectrometer. 


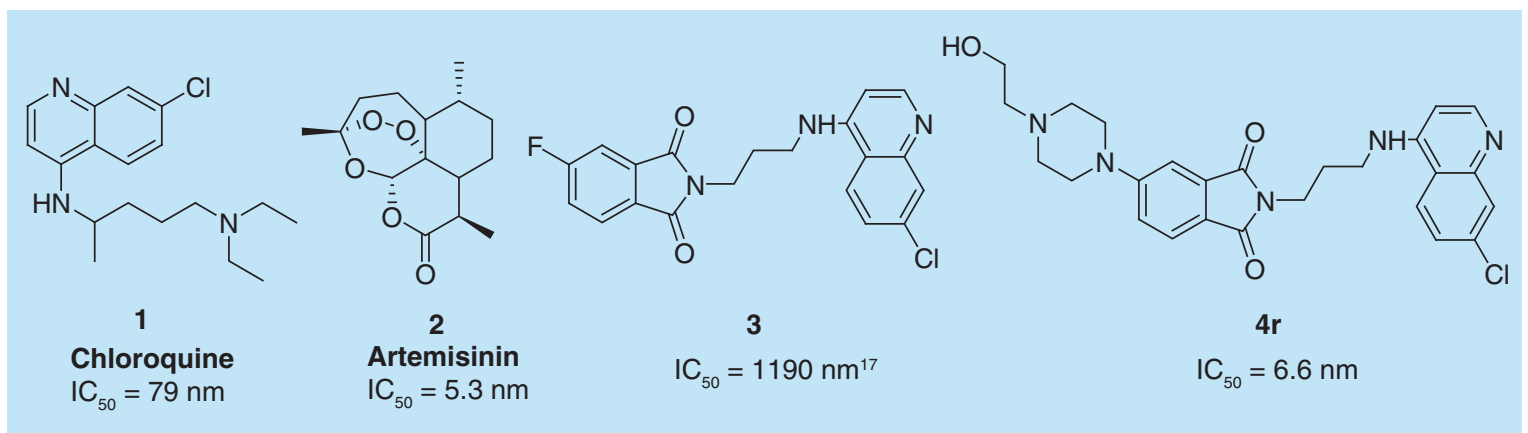

Figure 1. Structure of well-known antimalarial drugs 1, 2, the previously reported 2-(3-[7-chloroquinolin-4-ylamino]propyl)-5-fluoroisoindoline-1,3-dione 3 and newly synthesized (4r) hydroxyethylpiperazine-isoindoline-1,3-dione-4-aminoquinoline with their antiplasmodial activity against the chloroquine-resistant W2 strain of Plasmodium falciparum.

\section{Synthesis}

General procedure for the preparation of $\mathrm{C} 4 / \mathrm{C} 5$ cycloalkyl amine substituted isoindoline-1,3-dione-4-aminoquinolines (4a-t)

To a microwave reaction vial was added a solution of C-3/C-4 fluoro-phthalic anhydride $(1.0 \mathrm{mmol})$ in $0.5 \mathrm{ml}$ of $\mathrm{N}$-methylpyrrolidin-2-one (NMP) and 4-aminoquinoline-diamines $(1.0 \mathrm{mmol})$. After sealing with a PTFE cap, the vessel was heated to $130^{\circ} \mathrm{C}$ for $2 \mathrm{~min}$ in the microwave reactor. After the accomplishment of the first step, as obvious from TLC, cycloalkyl amine $(1.2 \mathrm{mmol})$ was added in the same reaction vial. The reaction mixture was again heated at $160^{\circ} \mathrm{C}$ for $5 \mathrm{~min}$ in the microwave reactor and the completion was ascertained using TLC. After completion, the contents were poured in water $(20 \mathrm{ml})$ resulting in the precipitation of the desired product. The obtained product was filtered and recrystallized using absolute ethanol.

\section{Methods for assessment of antiplasmodial activity of test compounds}

The W2 strain of $P$. falciparum was cultured in RPMI-1640 medium with 0.5\% Albumax I (Gibco, MA, USA), following standard methods, and parasites were synchronized with 5\% D-sorbitol. Beginning at the ring stage, microwell cultures were incubated with different concentrations of compounds for $48 \mathrm{~h}$. The compounds were added from DMSO stocks; the maximum concentration of DMSO used was $0.1 \%$. Controls without inhibitors included $0.1 \%$ DMSO. After $48 \mathrm{~h}$, when control cultures had progressed to new rings, the culture medium was removed, and cultures were incubated for $48 \mathrm{~h}$ with $1 \%$ formaldehyde in phosphate-buffered saline (PBS), $\mathrm{pH}$ 7.4, at room temperature. Fixed parasites were then transferred to $0.1 \%$ Triton X-100 in phosphate-buffered saline containing $1 \mathrm{nM}$ YOYO-1 dye (Molecular Probes, MA, USA). Parasitemia was determined from dot plots (forward scatter vs fluorescence) acquired on a FACSort flow cytometer using Cell Quest software (Beckton Dickinson, NJ, USA). IC $_{50}$ values for growth inhibition were determined from plots of percent control parasitemia over inhibitor concentration using the Prism 5.0 program (GraphPad Software, CA, USA), with data from duplicate experiments fitted by nonlinear regression.

\section{Cytotoxicity assay}

Cell viability was determined using Vero cells (ATCC, Sigma, Munich, Germany), which were grown in RPMI medium (Gibco) supplemented with 10\% decomplemented fetal calf serum under 5\% $\mathrm{CO}_{2}$ atmosphere. Cells were seeded in 96-well plates at a density of $2 \times 10^{4}$ cells/well in $160 \mu \mathrm{l}$ medium and incubated overnight at $37^{\circ} \mathrm{C}$ to allow cells to adhere. Compounds (dissolved in DMSO) were freshly diluted to appropriate concentrations in DMEM, to allow the addition of $20 \mu \mathrm{l}$ volumes of the diluted compounds to the cells that resulted in final compound concentrations of 100,50 and $25 \mu \mathrm{g} / \mathrm{ml}$. The maximum final concentration of DMSO was $1 \%$ (v/v); no cytotoxic effect of DMSO was observed at this concentration. After 24-h incubation at $37^{\circ} \mathrm{C}, 20 \mu \mathrm{l}$ of $1 \mathrm{mg} / \mathrm{ml}$ resazurin (Sigma) was added to each well and the cells were incubated for an additional $3 \mathrm{~h}$ at $37^{\circ} \mathrm{C}$. Fluorescence was measured in a Polarstar Omega fluorometer using appropriate filters $(540 \mathrm{~nm}$ excitation and $590 \mathrm{~nm}$ emission wavelength). Percentage survival was determined by dividing fluorescence values obtained in the compound containing wells by values obtained for control wells containing cells incubated with a dilution series 


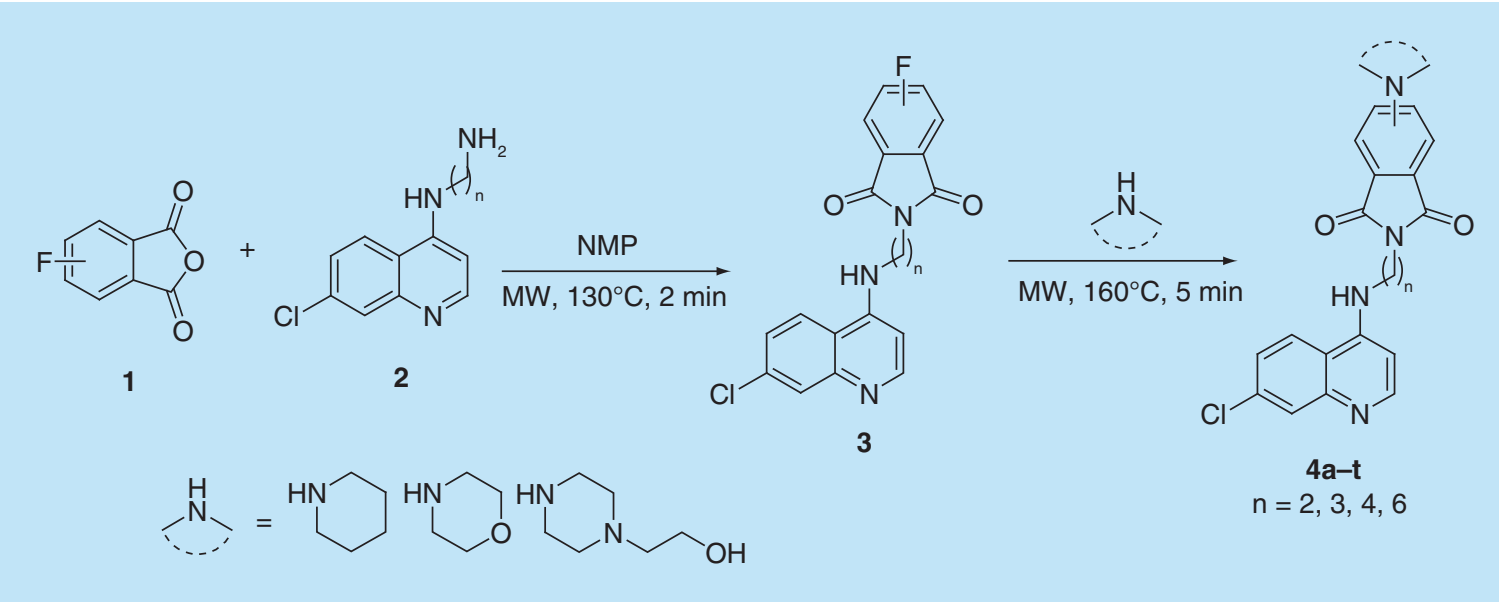

Figure 2. Microwave-promoted synthesis of C4/C5 cycloalkyl amino $\left(2^{0}\right)$ isoindoline-1,3-dione-4-aminoquinolines (4a-t).

MW: Molecular weight; NMP: N-methylpyrrolidin-2-one.

of DMSO $(1,0.5,0.25 \%)$ and multiplying this value by 100 . The $\mathrm{IC}_{50}$ was defined as the lowest concentration of compound tested at which at least $50 \%$ cell viability was observed.

\section{Results \& discussion}

\section{Chemistry}

The preparation of target cycloalkyl-amine linked isoindoline-1,3-dione-4-aminoquinolines was carried out using microwave heating as the reaction under conventional heating was sluggish. A range of solvents including dimethylformamide (DMF), dimethylsulfoxide (DMSO) and NMP was chosen for optimizing the reaction conditions and the best results were observed using NMP. Thus, the treatment of 3/4- fluoro-phthalic anhydride with $\mathrm{N}$-(7-chloroquinolin-4-yl) [31] based diamines in NMP at $130^{\circ} \mathrm{C}$ under microwave heating for 2 min led to the synthesis of corresponding 4-aminoquinoline-isoindolines 3 in good to excellent yields (74-92\%). The microwave heating of 3 with various cycloalkyl-based secondary amines in $\mathrm{NMP}$ at $160^{\circ} \mathrm{C}$ for 5 min led to the formation of C4/C5 cycloalkyl amine-linked isoindoline-1,3-dione-4-aminoquinolines (4a-t) in excellent yields (85-92\%) as elucidated in Figure 2.

The structures of the synthesized compounds were assigned on the basis of spectral data and analytical evidence. The compound 4r, for example, analyzed as $\mathrm{C}_{26} \mathrm{H}_{28} \mathrm{ClN}_{5} \mathrm{O}_{3}$, showed a molecular ion peak at $m / z 494.2160$ $[\mathrm{M}+\mathrm{H}]^{+}$in its High-Resolution Mass Spectrum. The salient features of its ${ }^{1} \mathrm{H}$ NMR spectrum included the appearance of multiplets at $\delta$ (pp.m.) 1.91-1.98, 2.23-2.28 and triplet at $3.62(\mathrm{~J}=6.7 \mathrm{~Hz})$ corresponding to methylene protons, triplets at $2.39(\mathrm{~J}=6.1 \mathrm{~Hz})$ and $3.50(\mathrm{~J}=6.2 \mathrm{~Hz})$ because of ethyl linked to piperazine, two triplets at $2.50(J=4.7 \mathrm{~Hz})$ and $3.33(J=4.5 \mathrm{~Hz})$ because of piperazine ring protons and a doublet at $\delta$ (p.p.m.) $7.54(J=8.5 \mathrm{~Hz})$ because of the aromatic proton of the isoindoline-dione ring. Its ${ }^{13} \mathrm{C}$ NMR spectrum revealed the appearance of absorptions at $\delta(\mathrm{ppm}) 168.3$ and 168.7, corresponding to isoindoline-dione carbonyls, along with methylene carbons at $\delta 27.3,35.8,40.6,47.4$ and 53.2 and piperazine carbons at $\delta 59.0$ and 60.5 p.p.m., as confirmed by ${ }^{13} \mathrm{C}$ NMR (DEPT) spectrum. Further $1 \mathrm{H}-1 \mathrm{H}$ COSY and ${ }^{1} \mathrm{H}_{-}{ }^{13} \mathrm{C}$ HSQC (Supplementary data) correlations substantiated the assigned structure.

\section{Antiplasmodial evaluation \& SARs}

The synthesized C4/C5substituted-isoindoline-1,3-diones-4-aminoquinolines were screened for their antiplasmodial activity against CQR (W2) strain of P. falciparum (Table 1). Most of the compounds displayed good antiplasmodial activities, with $\mathrm{IC}_{50} \mathrm{~s}$ in the low nanomolar range. The antiplasmodial activities of synthesized scaffolds depended on the nature of the substituent at the C4/C5 positions of the isoindoline-1,3-dione as well as on the length of the alkyl chain (n) introduced as a spacer. Analyzing the SAR among $\mathbf{4 a}-\mathbf{d}(\mathrm{R}=$ piperidyl, C4), an increase in chain length resulted in improvement in antiplasmodial activities, with compounds $4 \mathbf{c}(\mathrm{n}=4)$ and $4 \mathbf{d}(\mathrm{n}=6)$ with $\mathrm{IC}_{50} \mathrm{~s}$ of 0.05 and $0.075 \mu \mathrm{M}$, respectively. Changing the position of piperidine from C4 to C5 
Table 1. Antiplasmodial activities, cytotoxic sevaluation on the mammalian Vero cells and their selectivity index of tested compounds against chloroquine-resistant W2 strains of Plasmodium falciparum.

\begin{tabular}{lllllll} 
Code & Structure & $\mathrm{IC}_{50}(\mu \mathrm{M})$ & $\mathrm{IC}_{50}(\mu \mathrm{M}$ cytotoxicity) & $\mathrm{SI}$ & $\mathrm{Clog}^{\dagger}$ & $\mathrm{Clog}^{\dagger} \mathrm{P}(\mathrm{p})$ \\
\hline $4 \mathrm{a}$ & & 0.158 & $<28.80$ & 182 & 4.55 & 1.39
\end{tabular}<smiles>O=C1c2cccc(N3CCCCC3)c2C(=O)N1CCNc1ccnc2cc(Cl)ccc12</smiles>

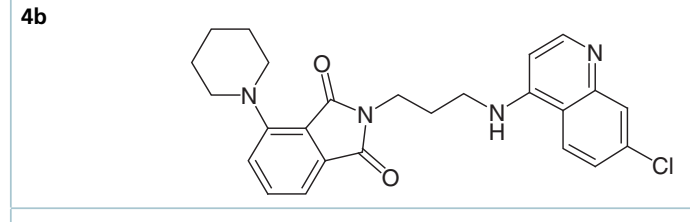

0.162

55.80

344.4

4.82

1.66

4c<smiles>O=C1c2cccc(N3CCCCC3)c2C(=O)N1CCCCNc1ccnc2cc(Cl)ccc12</smiles>

0.051

54.10

1060.7

5.09

1.93

4d 0.075

204.00

2720

6.10

2.94<smiles>O=C1c2cccc(N3CCCCC3)c2C(=O)N1CCCCCCNc1ccnc2cc(Cl)ccc12</smiles>

$4 e$ 0.163 $<28.80$ 177.7 4.88 1.72<smiles>O=C1c2ccc(N3CCCCC3)cc2C(=O)N1CCNc1ccnc2cc(Cl)ccc12</smiles>

$4 f$ 0.176 55.80 317 5.15 2.00<smiles>CC(C)(C)C</smiles>

49

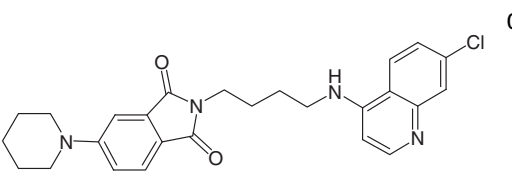
0.077 $<27.00$ 350.6 5.42 2.27

4h

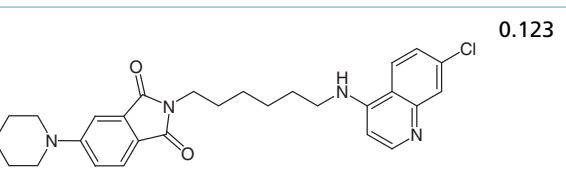

0.123

$<25.50$

207.3

6.43

3.28 
Table 1. Antiplasmodial activities, cytotoxic sevaluation on the mammalian Vero cells and their selectivity index of tested compounds against chloroquine-resistant W2 strains of Plasmodium falciparum (cont.).

$\begin{array}{lllllll}\text { Code } & \text { Structure } & \mathrm{IC}_{50}(\mu \mathrm{M}) & \mathrm{IC}_{50}(\mu \mathrm{M} \text { cytotoxicity) } & \mathrm{SI} & \mathrm{Clog} \mathrm{P}^{\dagger} & \mathrm{Clog} \mathrm{P}(\mathrm{p}) \\ \mathbf{4 i} & & 0.18 & <28.60 & 158.8 & 3.48 & 0.33\end{array}$<smiles>O=C1c2cccc(N3CCOCC3)c2C(=O)N1CCNc1ccnc2cc(Cl)ccc12</smiles>

$4 \mathbf{j}$<smiles>O=C1c2cccc(N3CCOCC3)c2C(=O)N1CCCNc1ccnc2cc(Cl)ccc12</smiles>

$\begin{array}{lllll}0.276 & 222.20 & 805 & 3.75 & 0.59\end{array}$

$4 \mathrm{k}$<smiles>O=C1c2cccc(N3CCOCC3)c2C(=O)N1CCCCNc1ccnc2cc(Cl)ccc12</smiles>

0.071

107.70

1517

4.03

0.87

An


Table 1. Antiplasmodial activities, cytotoxic sevaluation on the mammalian Vero cells and their selectivity index of tested compounds against chloroquine-resistant W2 strains of Plasmodium falciparum (cont.).

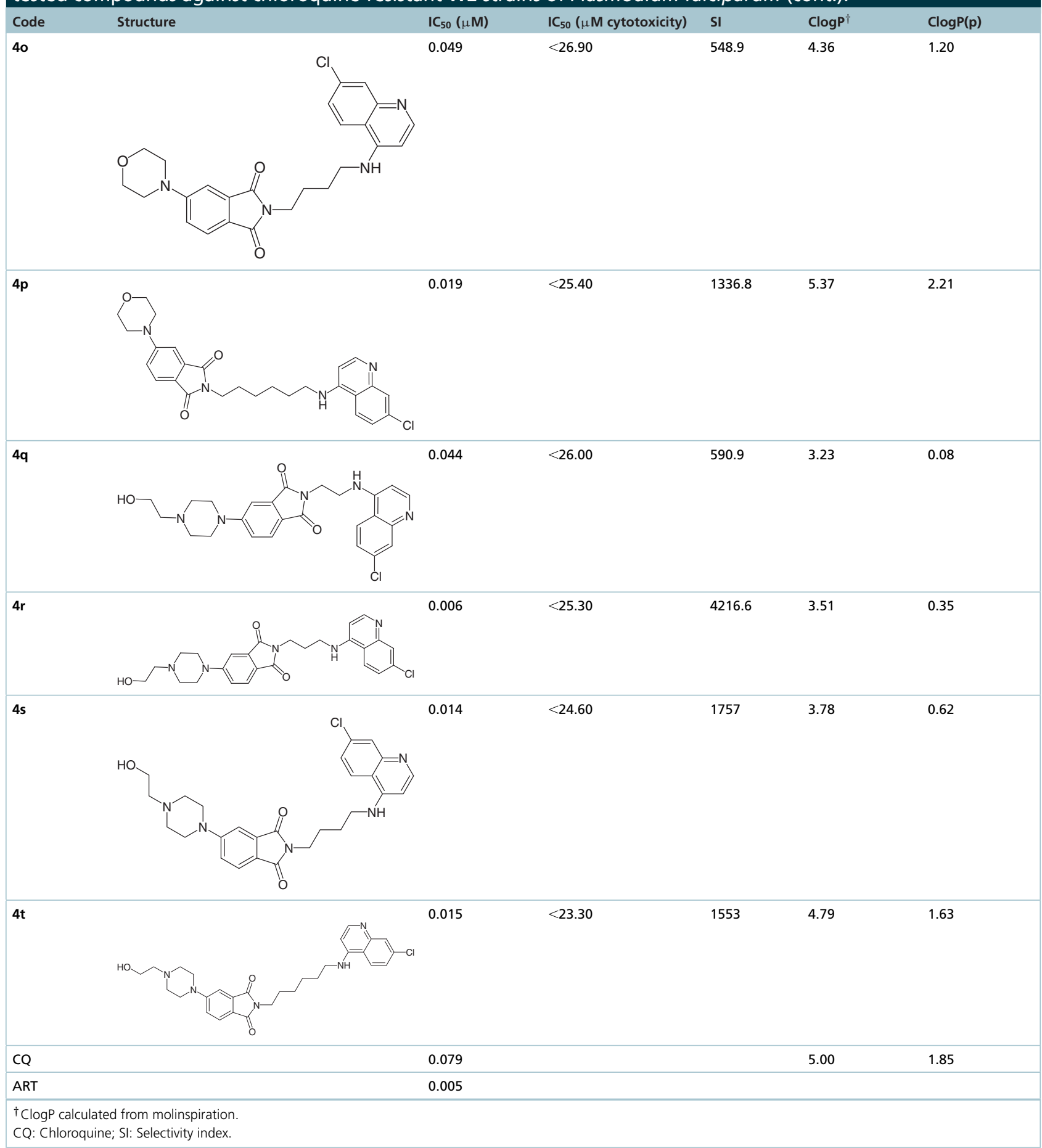

on the isoindoline-dione did not influence their antiplasmodial activities, as evidenced by compounds $\mathbf{4 e - h}$, with activities comparable with those of $\mathbf{4 a}-\mathbf{d}$. Replacing the piperidine with morpholine at the C4 position resulted in enhancement of activities, with compound $\mathbf{4 l}(\mathrm{n}=6)$ displaying an $\mathrm{IC}_{50}$ of $0.025 \mu \mathrm{M}$. The shifting of the morpholine ring from the $\mathrm{C} 4$ to $\mathrm{C} 5$ position further improved activity, with compound $\mathbf{4 p}$ exhibiting an $\mathrm{IC}_{50}$ of $0.019 \mu \mathrm{M}$. The introduction of hydroxyethyl-piperazine substituents on isoindoline-dione resulted in activities independent of the length of the spacer, as evidenced by $\mathbf{4 r}, \mathbf{4 s}$ and $\mathbf{4 t}$. Compound $\mathbf{4 r}$, with an optimum combi- 


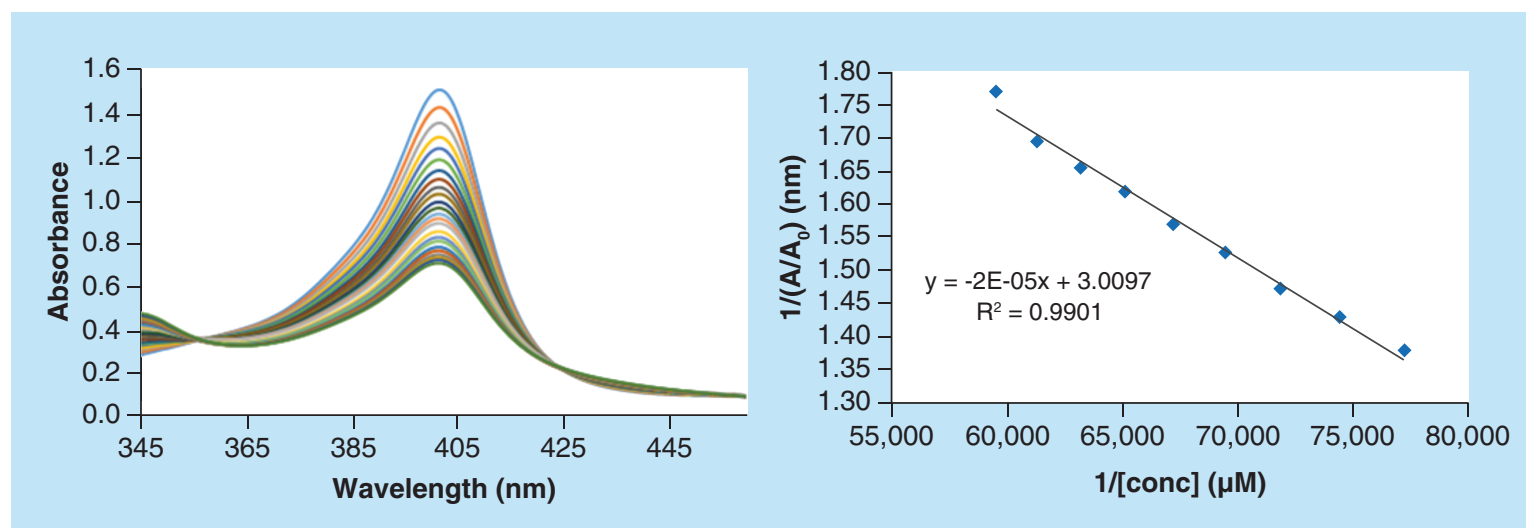

Figure 3. Titration of monomeric heme $(12 \mu \mathrm{M})$ at pH $7.4(0.02 \mathrm{M}$ HEPES buffer in $40 \%$ aq. DMSO solution) with an increasing concentration of $4 \mathrm{r}(0-18 \mu \mathrm{M}$ in $0.02 \mathrm{M}$ HEPES buffer in aqueous DMSO. Inset: plot of A401 nm vs concentration of $\mathbf{4 r}$.

Table 2. Binding constants $(\log K)$ for $4 \mathrm{r}$ and chloroquine.

\begin{tabular}{llc} 
Compound & \multicolumn{1}{c}{ Monomeric Heme log $K$} \\
\cline { 2 - 3 } & pH 5.6 (MES buffer) & pH 7.4 (HEPES buffer) \\
\hline CQ & 5.41 & 5.14 \\
\hline CQ: Chloroquine; HEPES: (4-(2-hydroxyethyl)-1-piperazine-1-ethanesufonic acid); MES: (2-(N-morpholino)ethanesulfonic acid). & 5.10 \\
\hline
\end{tabular}

nation of hydroxyethyl-piperazine at the $\mathrm{C} 5$ position and a propyl chain as a spacer, proved to be the most potent among the series, with an $\mathrm{IC}_{50}$ of $0.006 \mu \mathrm{M}$ (Figure 1).

To ascertain if the observed activities of synthesized scaffolds are due to their inherent antiplasmodial nature or to cytotoxicity, the compounds were evaluated against the mammalian VERO cell line (Table 1). The synthesized compounds were low to moderately cytotoxic, with good selectivity indices, especially $\mathbf{4 r}$ and $\mathbf{4 s}$, with selectivity indices of 4216 and 1757, respectively.

\section{Mode of action}

Heme-binding studies

4-aminoquinoline based antimalarials exert their antiplasmodial effect by complexation with $\mathrm{Fe}^{3+}$ ferriprotoporphyrin IX (FPIX), thus inhibiting the polymerization of heme to hemozoin. The heme inhibition occurs via $\pi-\pi$ stacking interactions of the quinoline ring with the porphyrin ring of FPIX. Considering the promising antiplasmodial activities of the synthesized series of compounds, we carried out heme-binding studies of the most active scaffold to decipher its mechanism of action [32]. In order to rationalize the binding mode, the interaction of $\mathbf{4 r}$ with hemin chloride was studied via spectrophotometric titrations at $\mathrm{pH} 7.4$ (physiological $\mathrm{pH}$ ) and 5.6 (approximate $\mathrm{pH}$ in the food vacuole of the parasite). Small increments of $4 \mathbf{r}(0-18 \mathrm{mM}, 40 \%$ DMSO) were added stepwise into a constant concentration of monomeric heme $(12 \mu \mathrm{M})$ at physiological $\mathrm{pH}(0.02 \mathrm{M}$ HEPES buffer in aqueous DMSO at $\mathrm{pH}$ 7.4) and acidic $\mathrm{pH}$ (0.02M MES buffer in aqueous DMSO at $\mathrm{pH}$ 5.6), resulting in a substantial decrease in intensity of the $\mathrm{Fe}(\mathrm{III})$ PPIX Soret band at $401 \mathrm{~nm}$, with no shift in the absorption maximum (Figures $3 \& 4$ ). Solvent (DMSO) had no effect on the binding of $\mathbf{4 r}$ with heme at either $\mathbf{p H}$ used in this experiment (Supplementary Figure 8). The binding constants (Table 2) were calculated by analyzing the titration curves using HypSpec, a nonlinear least-squares fitting program. Titrations of CQ with heme were performed under identical conditions, and binding constants were calculated for comparison purposes (Supplementary Figure 6-7). The binding constants for the complexes formed between monomeric heme and $\mathbf{4 r}$ were comparable with those of the standard antimalarial drug CQ at physiological and acidic $\mathrm{pH}$ (Table 2).

${ }^{1} \mathrm{H}$ NMR titrations were further carried out to confirm the binding of $\mathbf{4 r}$ with monomeric heme. The titration experiment was performed by recording the ${ }^{1} \mathrm{H}$ NMR spectrum of $\mathbf{4 r}$ in $40 \%$ DMSO- $\mathrm{d}_{6}: \mathrm{D}_{2} \mathrm{O}(10 \mu \mathrm{l})$ after the addition of heme $\left(10\right.$ and $20 \mathrm{~mol} \%$ ) dissolved in DMSO- $\mathrm{d}_{6}$. A minor shift in the aromatic and aliphatic 


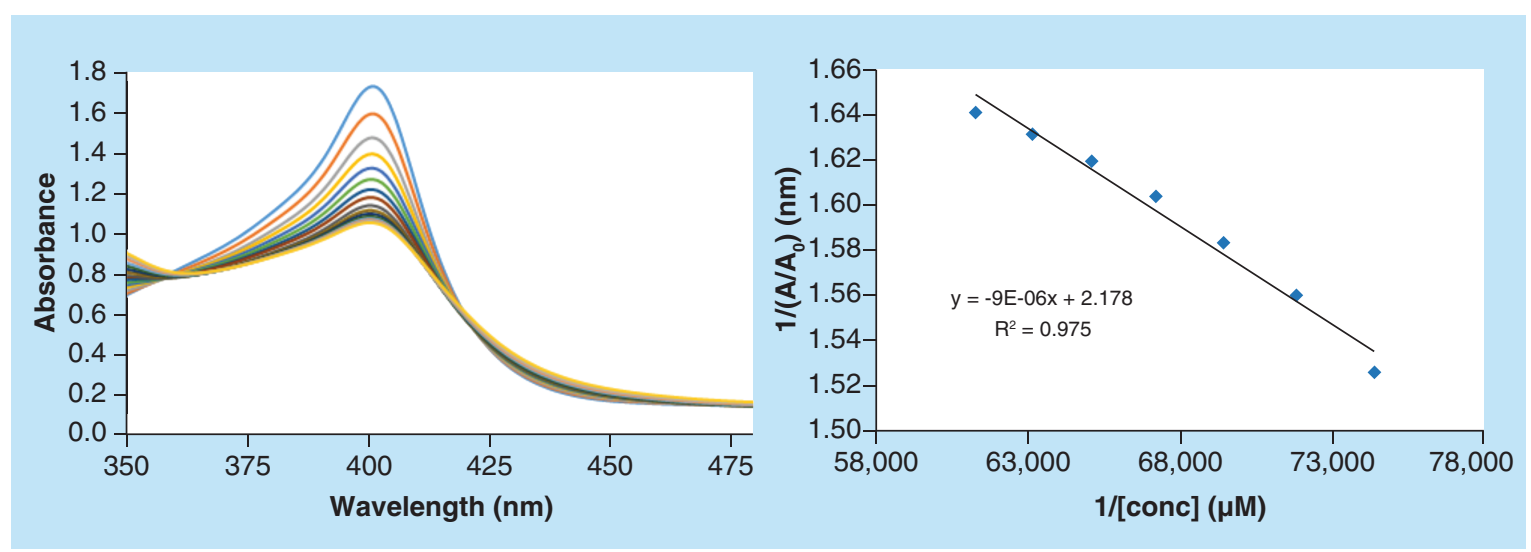

Figure 4. Titration of monomeric heme $(12 \mu \mathrm{M})$ at pH $5.6(0.02 \mathrm{M}$ MES buffer in $40 \%$ aq. DMSO solution) with an increasing concentration of $4 \mathrm{r}(0-16 \mu \mathrm{M}$ in $0.02 \mathrm{M}$ MES buffer in aqueous DMSO solution). Inset: plot of A401 $\mathrm{nm}$ vs concentration of $\mathbf{4 r}$.

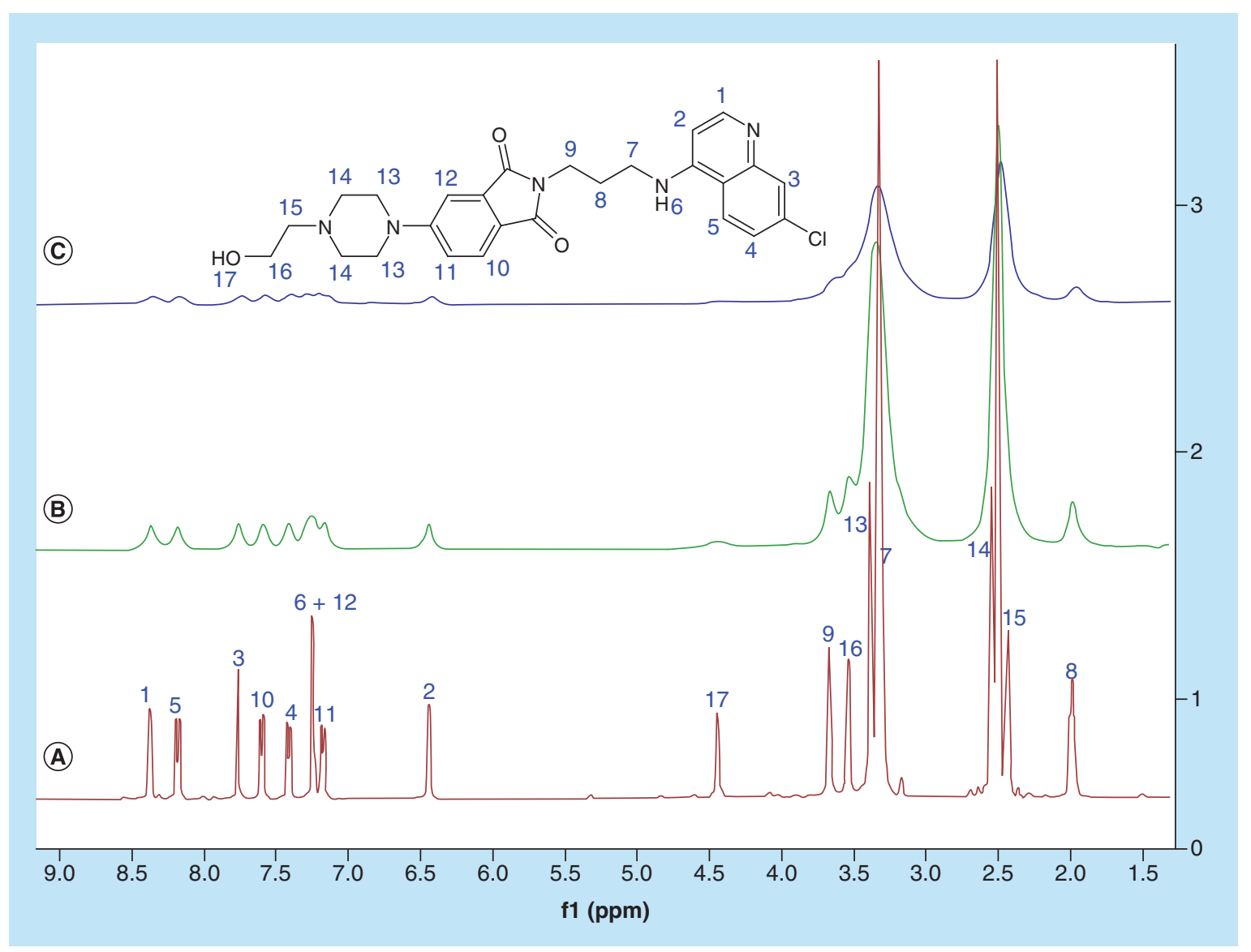

Figure 5. ${ }^{1} \mathrm{H}$ NMR spectral changes observed for $4 \mathrm{r}$ after addition of increasing amounts of heme: (A) $0 \mathrm{~mol} \%$, (B) $10 \mathrm{~mol}$ and (C) $20 \mathrm{~mol}$ in 40\% DMSO-d $\mathrm{d}_{6}: \mathrm{D}_{2} \mathrm{O} / \mathrm{D}_{2} \mathrm{SO}_{4}(10 \mu \mathrm{l})[\Delta \delta$ for peak: $1=0.01,2=0.00365,3=0.01,4=0.0185$, $e=0.00675, f(N H)=0.0264, g=0.0138, h=0.001,(\mathrm{OH})=0.0057, i=0.0113, j=0.00104, n=0.011$ p.p.m. $]$.

absorption peaks of $\mathbf{4 r}$ (Figure 5) was observed upon the addition of $10 \mathrm{~mol} \%$ of heme to the solution, indicating the binding of $\mathbf{4 r}$ with heme. However, further addition of heme led to the broadening of the peaks due to the paramagnetic effect of the Fe(III) of FPIX. Further, the mass spectral analysis of an equimolar ( $5 \mu \mathrm{mol})$ solution of hemin chloride and $\mathbf{4 r}$ showed a molecular ion peak at 1111.8667 Da (Figure 6), corresponding to the molecular 


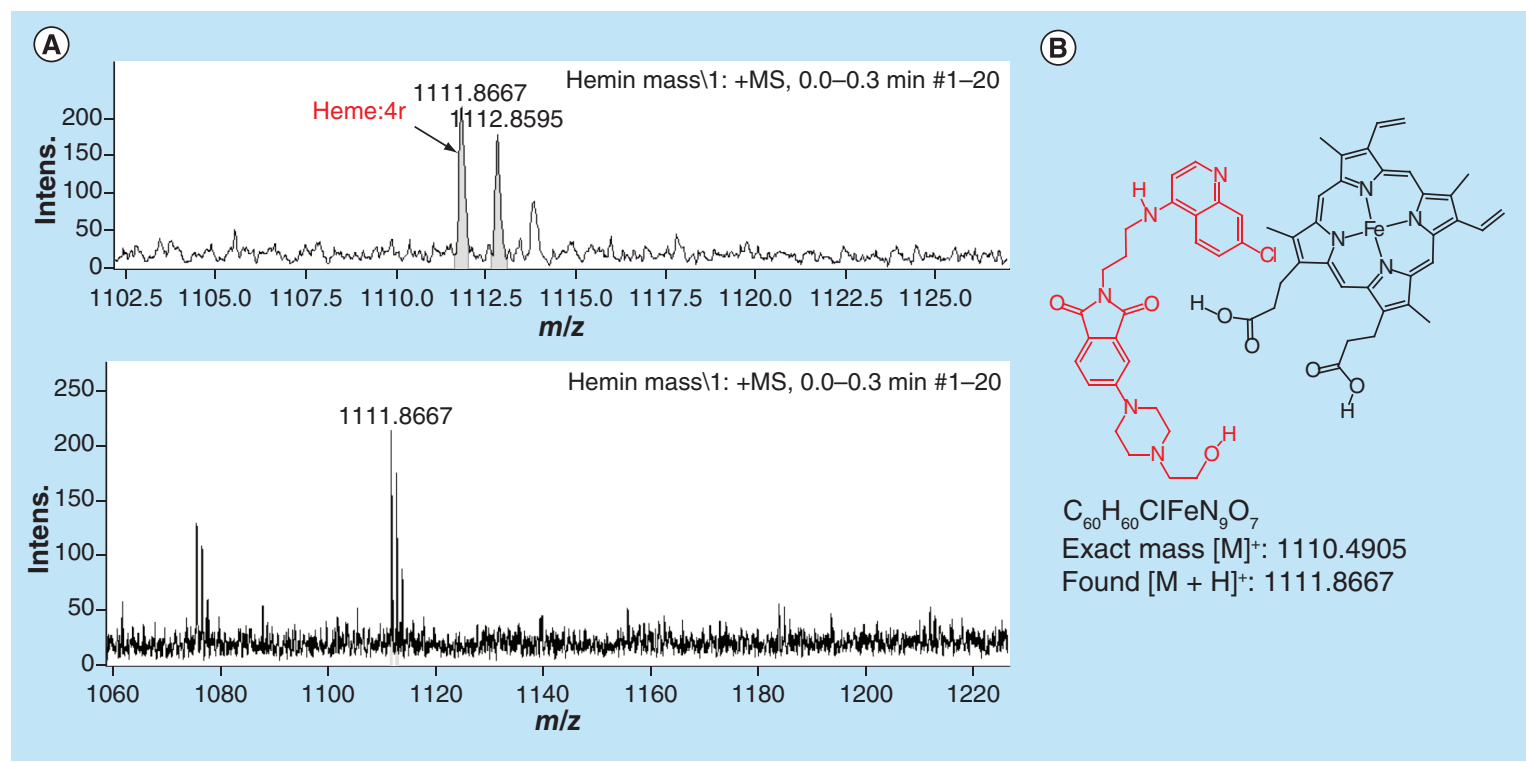

Figure 6. The solution phase spectra of $4 \mathrm{r}(5 \mu \mathrm{mol})$ upon addition of monomeric heme $(5 \mu \mathrm{mol})$ in $40 \%$ aq. DMSO solution.

formula $\mathrm{C}_{60} \mathrm{H}_{60} \mathrm{ClFeN}_{9} \mathrm{O}_{7}$, corroborating the formation of a 1:1 complex.

The heme-binding constant of $\mathbf{4 r}$ is almost identical to that of CQ while its activity is almost 12 -fold higher suggestive of the fact that the observed activity could not be explained purely on the basis of heme binding. The ClogP value of CQ is reported to be 5.0 [33], while the calculated $C \log P$ values of the promising compounds $\mathbf{4 r}, \mathbf{4 s}$ and $\mathbf{4 t}$ were found to be 3.51, 3.78 and 4.79, respectively (Table 1). Further, the protonation of the cycloalkyl amino group of the synthesized compound is feasible under physiological conditions. The ClogP values of protonated $\mathbf{4 r}, 4 \mathbf{s}$ and $4 \mathbf{t}$ were calculated to be $0.35,0.62$ and 1.63 which are quite appropriate from the perspective of bioavailability [34].

\section{Docking studies}

Two of the synthesized scaffolds $4 \mathbf{m}\left(\mathrm{IC}_{50}=0.370 \mu \mathrm{M}\right)$ and $\mathbf{4 r}\left(\mathrm{IC}_{50}=0.006 \mu \mathrm{M}\right)$ along with their protonated states $(\mathbf{4} \mathbf{m}[\mathbf{p}]$ and $\mathbf{4 r}[\mathbf{p}])$ were chosen for docking studies with heme. The best binding pose obtained for each ligand when all the protein catalytic residues around the heme are retained is further referred to as 'limited', while those obtained when all the catalytic residues are removed for the ligand to have complete access to heme catalytic surface is further referred to as 'free'. Results of the interaction of the ligand with heme as cofactor in the protein are shown in Supplementary Table 1.

Interestingly, both the molecules have better interaction with heme when in their di-protonated state as expected but the molecule $\mathbf{4 m}(\mathrm{p})$ has relatively better interaction than $4 \mathrm{r}(\mathrm{p})$ contrary to the experimental observation. The interaction energy with free-heme is characterized by significantly lower binding energy than the limited-heme.

\section{Interaction energy using the density functional theory method}

The interaction energy of each ligand with Hemin, Hematin and diHematin were computed after a partial optimization of each of the complexes as discussed in the computational method [33,35]. The results are shown in Supplementary Table 2.

The interaction of ligands with the limited surface of the Hemin, Hematin and diHematin revealed the best interaction between $\mathbf{4 r}(\mathbf{p})$ and Hemin $(-69.25 \mathrm{kcal} / \mathrm{mol}$, Supplementary Table 2) while the next in this series is the interaction of $\mathbf{4 m}(\mathbf{p})$ with Hematin $(-0.33 \mathrm{kcal} / \mathrm{mol}$, Supplementary Table 2$)$. All other interactions of the ligands with the limited surface are not favorable (positive values of energy). The interaction with the limited surface is the true representation of the catalytic mechanism of the Heme as described in the literature [35] which involves the proton transfer from the ligand via H... O...Fe interaction with the help of catalytic residue HIS 42 and ARG 38. $\mathbf{4 r}(\mathbf{p})$ does not only possess the best interaction but also assumes the catalytic mechanism of the H...O ...Fe interaction (Figure 7A) justifying it as a better inhibitor with $\mathrm{IC}_{50}$ value of $0.006 \mu \mathrm{M}$. 


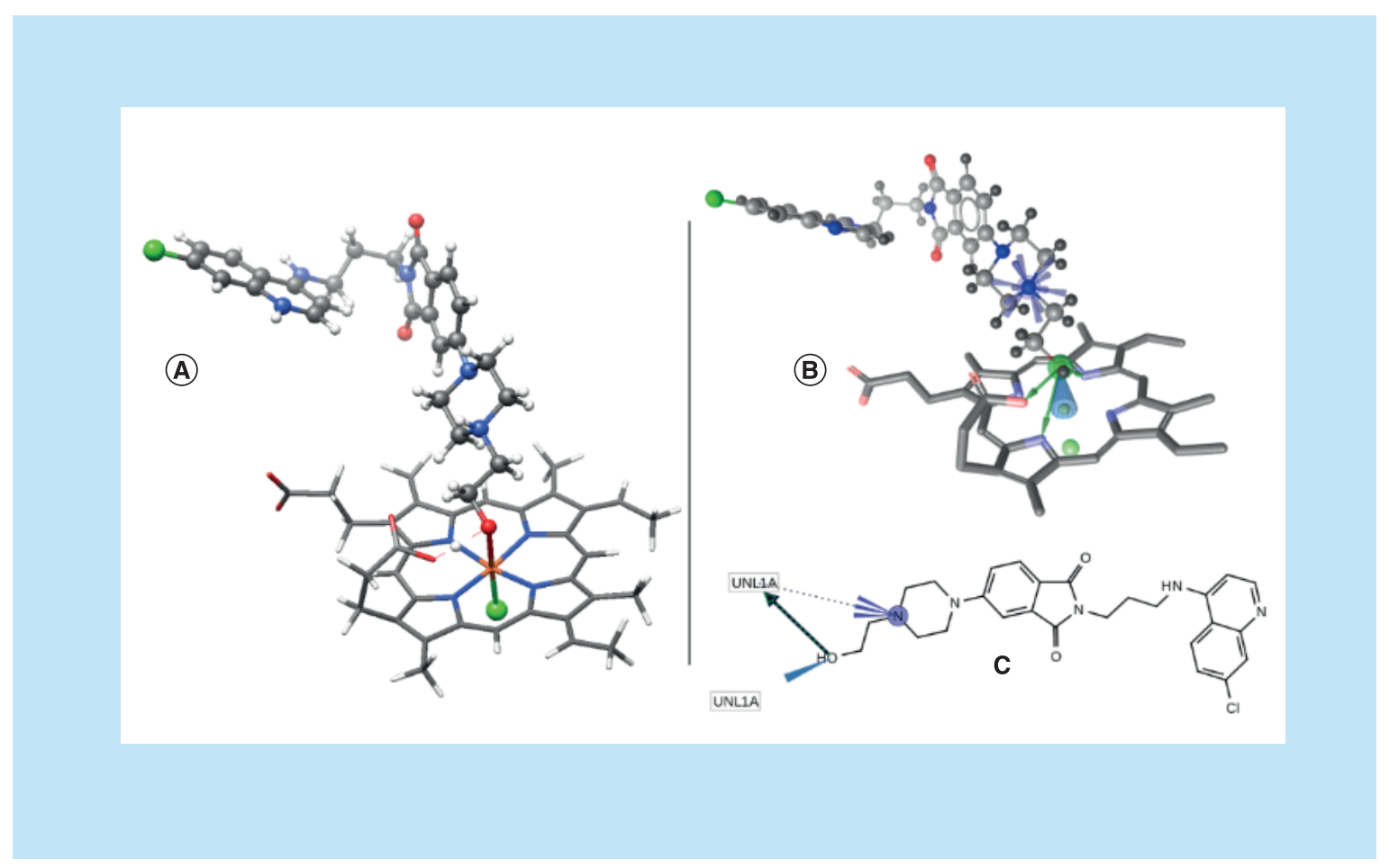

Figure 7. The interaction of $4 r(p)$ with Hemin showing the (A) geometry, pharmacophore in (B) 3D and (C) 2D.

The interaction of $\mathbf{4 r}(\mathbf{p})$ with the limited surfaces of Hemin is completely different from other ligand interactions. It is the only interaction that is associated with one positive ionizable, one iron-binding location, and three $\mathrm{H}$-bond donor interactions (Figure 7B \& C) while the interaction of $\mathbf{4 m}(\mathbf{p})$ with free Hemin and free Hematin (which is the best among the free surface interactions) are characterized mainly with four prominent hydrophobic interactions. The poor interaction of $\mathbf{4 m}(\mathbf{p})$ with free diHematin $(7.18 \mathrm{kcal} / \mathbf{m o l})$ is also characterized by four hydrophobic interactions.

Among all the interactions of the ligands with the limited surfaces, the interaction of $\mathbf{4 r}(\mathbf{p})$ with limited surface of Hemin has the highest charge transfer (0.235) while the highest values of electron transfer for free surfaces are obtained for the interaction of $\mathbf{4 m}(\mathbf{p})$ with free Hemin (0.564) and Hematin (0.557). Very poor values were obtained for the interactions of the ligands with diHematin since both HOMO and LUMO surface reside on the diHematin.

\section{Conclusion}

In summary, we report the synthesis and antiplasmodial evaluation of cycloalkyl amine isoindoline-1,3-dione-4aminoquinolines. Most of the compounds displayed good activity against CQR P. falciparum with low cytotoxicity and high selectivity indices. Among the synthesized compounds, $\mathbf{4 r}$ with an optimum combination of a propylchain as a linker and hydroxyethyl piperazine as the cycloalkyl amine was the most active with $\mathrm{IC}_{50} 0.006 \mu \mathrm{M}$ and a selectivity index $>4200$. The primary mode of action of these compounds appears to be binding with heme, as evident from heme-binding studies, further corroborated with NMR and high-resolution mass spectrum of the complex. The interaction energy of the diprotonated form of $\mathbf{4 r}(\mathbf{p})$ with heme models along with its favorable logP value could be the reason for its observed activity in comparison with that of CQ.

\section{Future perspective}

The recurrence of the isoindoline-1,3-dione ring as an important core, with some of its derivatives showing even higher biological effects than the well-known pharmacological molecules, has rejuvenated interest in this class of molecules. The promising antiplasmodial potential exhibited by the present series of C4/C5 cycloalkyl amine substituted isoindoline-1,3-dione-4-aminoquinolines is suggestive of the fact that these molecules can act as therapeutic templates for the design of new antimalarials with a low incidence of resistance. 


\section{Summary points}

- The battle against malaria is becoming a serious challenge due to the emergence of resistant strains of Plasmodium falciparum to currently employed antimalarials including artemisinin-based combination therapy.

- C4/C5 cycloalkyl amine substituted isoindoline-1,3-dione-4-aminoquinolines have been designed, synthesized and evaluated against the chloroquine-resistant strain of $P$. falciparum resulting in the identification of a potential lead.

- Heme-Inhibition and favorable partition coefficient value seemed to play a vital role in defining its mechanism of action.

Results

- C4/C5 cycloalkyl amine substituted isoindoline-1,3-dione-4-aminoquinolines were synthesized and bioevaluated for their antiplasmodial activity and cytotoxicity.

- The compounds with 2-hydroxyethyl-piperazine as secondary amine on the isoindoline-dione ring proved to exhibit good antiplasmodial profiles and were low to moderately cytotoxic to mammalian VERO cell line.

Conclusion

- The compound with an optimum combination of propyl chain length as a linker between two pharmacophores and 2-hydroxyethyl-piperazine as secondary amine on the isoindoline-dione ring proved to be the most potent among the series, exhibiting an $\mathrm{IC}_{50}$ value $0.006 \mu \mathrm{M}$ against chloroquine-resistant $\mathrm{W} 2$ strains of $P$. falciparum. Mechanistically, the compound exhibited an affinity toward Heme, as confirmed by UV-visible, HRMS and NMR titrations. The structure of the complex between the Heme and the ligand was further established using density functional theory studies.

\section{Supplementary data}

To view the supplementary data that accompany this paper please visit the journal website at: www.futurescience/doi/suppl/10.4155/fmc-2019-0260. Material and methods, Computational methods, density functional theory calculations, Synthesis and spectral data of all the compounds along with scanned ${ }^{1} \mathrm{H},{ }^{13} \mathrm{C},{ }^{13} \mathrm{C}-\mathrm{DEPT},{ }^{1} \mathrm{H}-{ }^{1} \mathrm{H}-\mathrm{COSY}$ and ${ }^{1} \mathrm{H}-{ }^{13} \mathrm{C}-\mathrm{HSQC}$ NMR spectra of few representative compounds viz. $\mathbf{4 c}, \mathbf{4 d}, \mathbf{4 f}, \mathbf{4 k}, \mathbf{4 m}, \mathbf{4 o}, \mathbf{4 r}$ and $\mathbf{4 s}$ are provided in the electronic supplementary information.

Financial \& competing interests disclosure

A Rani thanks CSIR, New Delhi, India, for providing financial assistance under CSIR-SRF Fellowship [09/254(0269)/2017-EMRI]. V Kumar acknowledges the Science and Engineering Research Board (SERB), New Delhi, for providing financial support [EMR/2015/001687]. We would also like to thank the Centre for High-Performance Computing based in Cape Town for access to computational resources. The authors have no other relevant affiliations or financial involvement with any organization or entity with a financial interest in or financial conflict with the subject matter or materials discussed in the manuscript apart from those disclosed.

No writing assistance was utilized in the production of this manuscript.

\section{References}

Papers of special note have been highlighted as: • of interest; $\bullet \bullet$ of considerable interest

1. Ashley EA, Phyo AP, Woodrow CJ. Malaria. Lancet 391(10130), 1608-1621 (2018).

2. Ridley RG. Medical need, scientific opportunity and the drive for antimalarial drugs. Nature 415(6872), 686-693 (2002).

3. Kumar N, Singh R, Rawat DS. Tetraoxanes: synthetic and medicinal chemistry perspective. Med. Res. Rev. 32(3), 581-610 (2012).

4. Hu YQ, Gao C, Zhang S et al. Quinoline hybrids and their antiplasmodial and antimalarial activities. Eur J. Med. Chem. 139, 22 (2017).

5. Bousema T, Drakeley C. Epidemiology and infectivity of Plasmodium falciparum and Plasmodium vivax gametocytes in relation to malaria control and elimination. Clin. Microbiol. Rev. 24(2), 377-410 (2011).

6. World malaria report 2017Alonso P, Bosman A, Cibulskis R et al.et al. (Eds). World Health Organization (2017). http://apps.who.int/iris/bitstream/10665/259492/1/9789241565523-eng.pdf?ua=1

7. World Malaria ReportAlonso P, Bosman A, Kolaczinski J et al.et al. (Eds). World Health Organization (2018). http://apps.who.int/iris/bitstream/handle/10665/275867/9789241565653-eng.pdf?ua=1

- Shows the current scenario (2018) of malaria, highlighting the critical issues of preventive and therapeutic measures.

8. Gemma S, Camodeca C, Brindisi M et al. Mimicking the intramolecular hydrogen bond: synthesis, biological evaluation, and molecular modeling of benzoxazines and quinazolines as potential antimalarial agents. J. Med. Chem. 55(23), 10387-10404 (2012).

9. Soares RR, de Silva JMF, Carlos BC et al. New quinoline derivatives demonstrate a promising antimalarial activity against Plasmodium falciparum in vitro and Plasmodium berghei in vivo. Bioorg. Med. Chem. Lett. 25(11), 2308-2313 (2015). 
10. Beteck RM, Smit FJ, Haynes RK, N’Da DD. Recent progress in the development of anti-malarial quinolones. Malar. J. 13, 339 (2014).

11. Ashley EA, Dhorda M, Fairhurst RM et al. Spread of artemisinin resistance in Plasmodium falciparum malaria. N. Engl. J. Med. 371(5), 411-423 (2014).

12. Das S, Saha B, Hati AK, Roy S, NJ. Evidence of artemisinin-resistant Plasmodium falciparum malaria in Eastern India. N. Engl. J. Med. 379(20), 1962-1964 (2018).

13. Das S, Manna S, Saha B, Hati AK, Roy S. Novel pfkelch13 gene polymorphism associates with artemisinin resistance in eastern India. Clin. Infect. Dis. 69(7), 1144-1152 (2019).

14. Meunier B. Hybrid molecules with a dual mode of action: dream or reality? Acc. Chem. Res. 41(1), 69-77 (2008).

15. Singh K, Kaur T. Pyrimidine-based antimalarials: design strategies and antiplasmodial effects. Med. Chem. Comm. 7(5), 749-768 (2016).

16. Ashley EA, Phyo AP. Drugs in development for malaria. Drugs 78(9), 861-879 (2018).

17. Kumar S, Bhardwaja TR, Prasad DN, Singh RK. Drug targets for resistant malaria: historic to future perspectives. Biomed. Pharmacother. 104, 8-27 (2018).

18. Biamonte BMA, Wanner J, Le Roch KG. Recent advances in malaria drug discovery. Bioorg. Med. Chem. Lett. 23(10), 2829-2843 (2013).

19. Bellot F, Cosledan F, Vendier L, Brocard J, Meunier B, Robert A. Trioxaferroquines as new hybrid antimalarial drugs. J. Med. Chem. 53(10), 4103-4109 (2010).

20. Kumar A, Srivastava K, Kumar SR et al. 4-anilinoquinoline triazines: a novel class of hybrid antimalarial agents. Eur. J. Med. Chem. 46(2), 676-690 (2011).

21. Manohar S, Rajesh UC, Khan SI, Tekwani BL, Rawat DS. Novel 4-aminoquinoline-pyrimidine based hybrids with improved in vitro and in vivo antimalarial activity. ACS Med. Chem. Lett. 3(7), 555-559 (2012).

22. Guantai EM, Ncokazi K, Egan TJ et al. Enone- and chalcone-chloroquinoline hybrid analogues: in silico guided design, synthesis, antiplasmodial activity, in vitro metabolism, and mechanistic studies. J. Med. Chem. 54(10), 3637-3649 (2011).

23. Sharma U, Kumar P, Kumar B. Recent advances in the chemistry of phthalimide analogues and their therapeutic potential. Mini Rev. Med. Chem. 10(8), 678-704 (2010).

-• Importance of phthalimides in Medicinal Chemistry.

24. Singh AK, Rajendran V, Pant A et al. Design, synthesis and biological evaluation of functionalized phthalimides: a new class of antimalarials and inhibitors of falcipain-2, a major hemoglobinase of malaria parasite. Bioorg. Med. Chem. 23(8), 1817-1827 (2015).

-• A comprehensive overview of new developments and insights in antiplasmodial research.

25. Rani A, Singh A, Gut J, Rosenthal PJ, Kumar V. Microwave-promoted facile access to 4-aminoquinoline-phthalimides: synthesis and anti-plasmodial evaluation. Eur. J. Med. Chem. 143, 150-156 (2018).

-• Methodology used in the synthesis of desired conjugates.

26. Rani A, Legac J, Rosenthal PJ, Kumar V. Substituted 1,3-dioxoisoindoline-4-aminoquinolines coupled via amide linkers: synthesis, antiplasmodial and cytotoxic evaluation. Bioorg. Chem. 88, 102912 (2019).

27. Singh A, Gut J, Rosenthal PJ, Kumar V. 4-aminoquinoline-ferrocenyl-chalcone conjugates: synthesis and anti-plasmodial evaluation. Eur. J. Med. Chem. 125, 269-277 (2017).

28. Rani A, Viljoen A, Johansen MD, Kremer L, Kumar V. Synthesis, anti-mycobacterial and cytotoxic evaluation of substituted Isoindoline-1,3-dione-4-aminoquinolines coupled via alkyl/amide linkers. RSC Adv. 9(15), 8515 (2019).

29. Singh A, Rani A, Gut J, Rosenthal PJ, Kumar V. Piperazine-linked 4-aminoquinoline-chalcone/ferrocenyl-chalcone conjugates: synthesis and antiplasmodial evaluation. Chem. Biol. Drug Des. 90(4), 590-595 (2017).

30. Kelly JX, Smilkstein MJ, Brun R et al. Discovery of dual function acridones as a new antimalarial chemotype. Nature 459(7244), 270-273 (2009).

31. De D, Krogstad FM, Byers LD, Krogstad DJ. Structure-activity relationships for antiplasmodial activity among 7-substituted 4-aminoquinolines. J. Med. Chem. 41(25), 4918-4926 (1998).

- Synthesis of precursor described in this manuscript.

32. Singh K, Kaur H, Smith P, Kock C de, Chibale K, Balzarini J. Quinoline-pyrimidine hybrids: synthesis, antiplasmodial activity, SAR, and mode of action studies. J. Med. Chem. 57(2), 435-448 (2014).

33. Sakata $Y$, Yabunaka K, Kobayashi $Y$ et al. Potent antimalarial activity of two arenes linked with triamine designed to have multiple interactions with heme. ACS Med. Chem. Lett. 9(10), 980-985 (2018).

34. Molinspiration. Calculation of molecular properties and bioactivity score (2019). www.molinspiration.com/cgi-bin/properties

35. Córdoba A, Magario I, Luján M. Experimental design and MM2-PM6 molecular modelling of hematin as a peroxidase-like catalyst in Alizarin Red S degradation. J. Mol. Catal. A. Chem. 355, 44-60 (2012).

- Methodology used for density functional theory calculations. 
